\title{
Intensity-modulated radiation therapy for permanent alopecia of unwanted palatal hair
}

\author{
Carryn Anderson • Ann Morris • John M. Buatti • \\ Deborah Alt $\cdot$ Rodrigo Bayon
}

Received: 3 May 2012 / Accepted: 21 June 2012 /Published online: 10 July 2012

(C) Springer-Verlag 2012

\begin{abstract}
Options for unwanted intraoral hair removal associated with flap reconstruction may include trimming, electrolysis, mechanical epilation, and laser and light epilation. These methods provide temporary relief and often require repeated treatment, with laser and light epilation providing the most durable response. Therapeutic external beam radiation used in the treatment of cancer is known to cause temporary alopecia at low doses and permanent alopecia at higher doses. We report a case of a man who underwent oncologic resection for early stage retromolar trigone cancer. His surgical defect required reconstruction with a submental flap. Post-operatively, his flap grew a thick beard within his oral cavity that was extremely bothersome. Due to the location of the flap, trimming, electrolysis, laser, and light epilation were not recommended treatment
\end{abstract}

This article is accepted for Poster Presentation at the 8th International Conference on Head and Neck Cancer, July 2012, Toronto, Ontario, Canada.

C. Anderson $(\bowtie) \cdot$ A. Morris $\cdot$ J. M. Buatti

Department of Radiation Oncology,

University of Iowa Hospitals and Clinics,

01614 PFPW, 200 Hawkins Dr,

Iowa City, IA 52242, USA

e-mail: carryn-anderson@uiowa.edu

\section{A. Morris}

e-mail: ann-morris@uiowa.edu

J. M. Buatti

e-mail: john-buatti@uiowa.edu

\section{R. Bayon}

Department of Otolaryngology,

University of Iowa Hospitals and Clinics,

Iowa City, IA, USA

e-mail: rodrigo-bayon@uiowa.edu

D. Alt

Department of Otolaryngology, Iowa City VA Health Care System, Iowa City, IA, USA

e-mail: deborah.alt@va.gov options. He was referred to us for consideration of external beam radiation with the goal of permanent alopecia of his intraoral graft.

Keywords Palatal hair · Radiation-induced alopecia $\cdot$ Hair removal $\cdot$ Oral cavity reconstruction

\section{Case report}

A 71-year-old smoker presented with a pedicled mass on the left hard palate extending behind the maxillary gingiva, down the retromolar trigone to the posterior mandibular buccal sulcus, and onto the buccal surface. Biopsy confirmed a spindled squamous cell carcinoma. CT scan showed a $2.5 \times 2.0 \mathrm{~cm}$ mass of the left posterior roof of the oral cavity. PET/CT scan confirmed uptake in the hard palate and left retromolar trigone with two hypermetabolic lymph nodes in level II. His past medical history included lung cancer treated with resection and adjuvant radiation 7 years prior, chronic obstructive pulmonary disease with oxygen dependence at night, and recurrent pneumonias. He had a $100+$ pack-year smoking history and quit at the time of his diagnosis.

Oncologic resection was performed, with pathology revealing two separate primaries. The first lesion was a $3.8-\mathrm{cm}$ spindle cell squamous carcinoma of the hard palate with a depth of invasion of $2 \mathrm{~mm}$, no bone involvement, no perineural invasion, and negative margins. The diameter of the pedicle of the mass was smaller than maximum diameter. The second lesion was a $1.5 \mathrm{~cm}$ moderately differentiated squamous cell carcinoma of the retromolar trigone with a depth of invasion of $2 \mathrm{~mm}$, no bone invasion, and negative margins. Due to the proximity of the presenting lesions, they were resected in a single en bloc specimen. The ipsilateral neck was dissected and pathologically negative (0/14 lymph nodes). The resulting 
intraoral defect required reconstruction with a submental flap rotated into the retromolar/palatal wound.

The patient's case was presented at multidisciplinary tumor board. Given the favorable pathologic features of each lesion taken individually, as well as negative margin and negative dissected neck, the oncologic benefit of adjuvant radiation was felt to be minimal. Due to his smoking and lung cancer history, he would be at high risk for additional primary malignancies where radiation might be necessary in the future. This discussion was brought to the patient and both adjuvant radiation and careful observation were discussed as options to consider. He chose careful observation. He remained without evidence of disease on active surveillance, however, he struggled with profuse hair growth on his oral cavity submental tissue graft. This resulted in a constant foreign body sensation and occasional dysphagia. Dermatology could not perform effective electrolysis or laser hair removal due to the location of the graft. Therefore, palliative radiation was considered as a potential solution.

At the time of radiation oncology consultation 5 months after his oncologic resection, his examination revealed thick intraoral hair growth on the graft along the left buccal mucosa and soft palate (Fig. 1). There was no clinical evidence of recurrent cancer. After discussion of the acute and latent risks of using radiation for this benign condition, including secondary malignancy and possible limitation of radiation as a treatment modality for future head and neck malignancy, the patient wished to proceed.

Simulation was in the supine position with a head and shoulder Aquaplast mask used for immobilization. Contrastenhanced CT and contrast-enhanced MRI were performed in the immobilization mask. These, together with his physical examination, informed the contouring of the intraoral graft and the design of the clinical target volume (CTV). A 3-mm three-dimensional expansion of the CTV was used for the planning target volume (PTV). The contralateral oral cavity was contoured as an avoidance structure. An intensity- modulated radiation therapy (IMRT) plan was created to deliver $50 \mathrm{~Gy}$ in 25 fractions to the PTV (Fig. 2). Mean dose to the left and right parotid was 15.5 and $14 \mathrm{~Gy}$, respectively. Spinal cord maximum point dose was 19.7 Gy. Daily conebeam CT was performed to optimize patient positioning.

By treatment visit at $18 \mathrm{~Gy}$, the patient noticed decreased hair on his graft. At $26 \mathrm{~Gy}$, virtually all the hair was gone. At completion of $50 \mathrm{~Gy}$, he had mild mucositis but maintained his weight on a full oral diet. His taste was slightly altered and he had minimal dry mouth. He had mild skin erythema.

At follow-up 1 month after completion of radiation, there was almost complete alopecia of the graft. The patient felt as though his taste had almost returned to normal and he denied any significant dryness. The radiation mucositis and dermatitis had resolved.

Ten months after his oncologic resection, he had a PET/CT scan for oncologic surveillance and this was negative for disease. At 8 months after completion of radiation (14.5 months after resection), he continued to have mild xerostomia managed with extra fluids. His taste and swallowing were normal and there was no trismus. On examination, there was no evidence of hair on the graft and no clinical evidence for cancer recurrence (Fig. 3).

\section{Discussion}

Intraoral hair growth is a known consequence of complicated tissue reconstruction procedures after oncologic resection in the oral cavity and oropharynx [1]. If an oncologic resection is extensive enough that it requires free-flap or rotational flap reconstruction, it often heralds locally advanced cancer that requires adjuvant therapeutic radiation to prevent cancer recurrence. The adjuvant radiation (generally 54-66 Gy fractionated over 5.5-7 weeks) subsequently causes permanent and complete alopecia of the graft tissue. Badran et al. histologically compared radiated versus non-irradiated oral cavity radial forearm free flaps, revealing
Fig. 1 Thick intraoral hair growth on the graft along the left buccal mucosa and soft palate
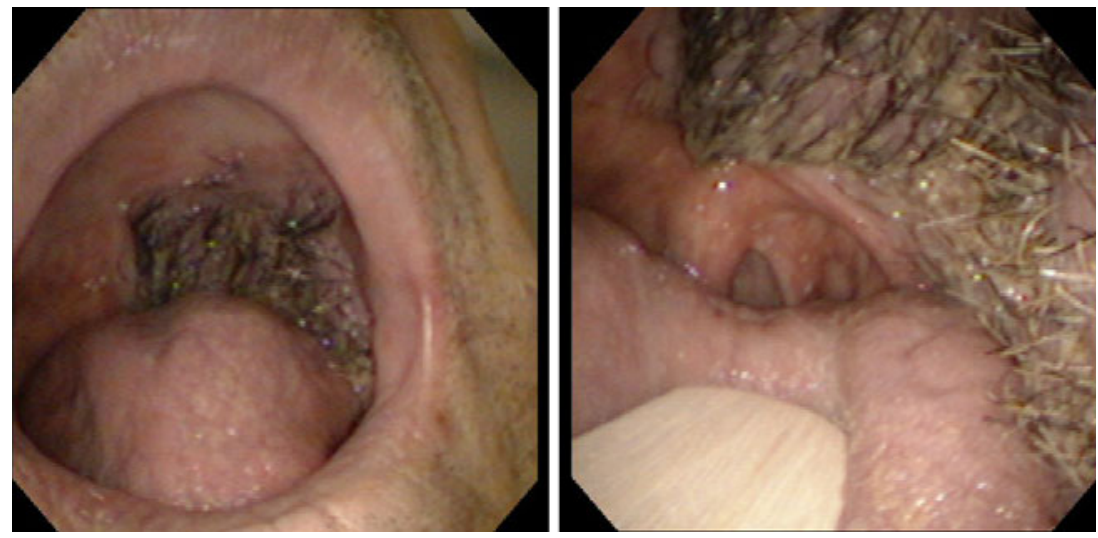
Fig. 2 An IMRT plan was created to deliver 50 Gy in 25 fractions. The planning target volume (PTV) is displayed in red. The 50 Gy (yellow), 40 Gy (green), and $30 \mathrm{~Gy}$ (blue) isodose lines are displayed. Bilateral parotids (left purple, right pink) and spinal cord (orange) are spared from significant radiation exposure
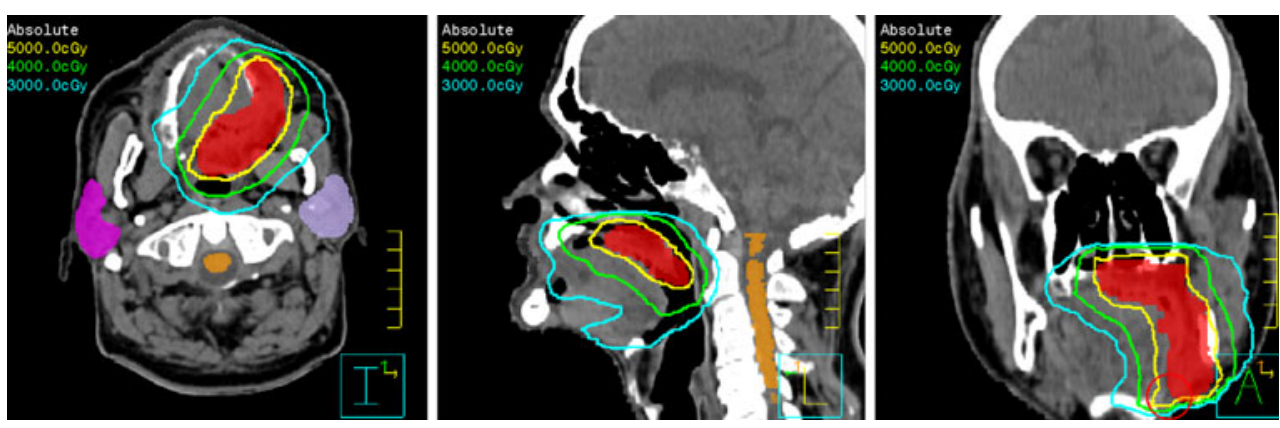

[4]. Three courses of treatment at 6-week intervals resulted in permanent alopecia as measured at 6 months after completion of therapy. Disadvantages of the technique included the bulky hand piece of the instrument making application in the oropharynx difficult and a reported paradoxical stimulation of hair growth in $10 \%$ of patients [5].

Laser epilation would have been the recommended treatment option for this patient. Dermatology assessed him for the procedure and determined they would not be able to reach the most posterior aspect of the graft, which was the source of his globus sensation and dysphagia. We are not advocating IMRT as a routine alternative to epilation, but an option in rare cases where epilation is not feasible and the potential benefits as discussed and considered by the patient outweigh the risks.

This patient's case was considered carefully, being mindful of his age, smoking status, medical comorbidities, the acute and late side effects caused by an external beam radiation course, including the risks of xerostomia, dysgeusia, dysphagia, second malignancy, and limitation of future use of radiation for oncologic purposes. Contrast-enhanced $\mathrm{CT}$ and MRI images were used for radiation simulation to contour the graft and normal tissues with the greatest possible accuracy. The patient was immobilized with a head and shoulder Aquaplast mask and a daily conebeam CT assisted in optimizing positioning to allow tighter margins. These techniques together with an IMRT delivery resulted in the lowest possible exposure of normal tissues.

The decision to deliver 50 Gy in 25 fractions was influenced by the work published by Lawenda et al. [6]. In their
Fig. 3 Follow-up at 8 months shows the patient has permanent, complete alopecia of the graft
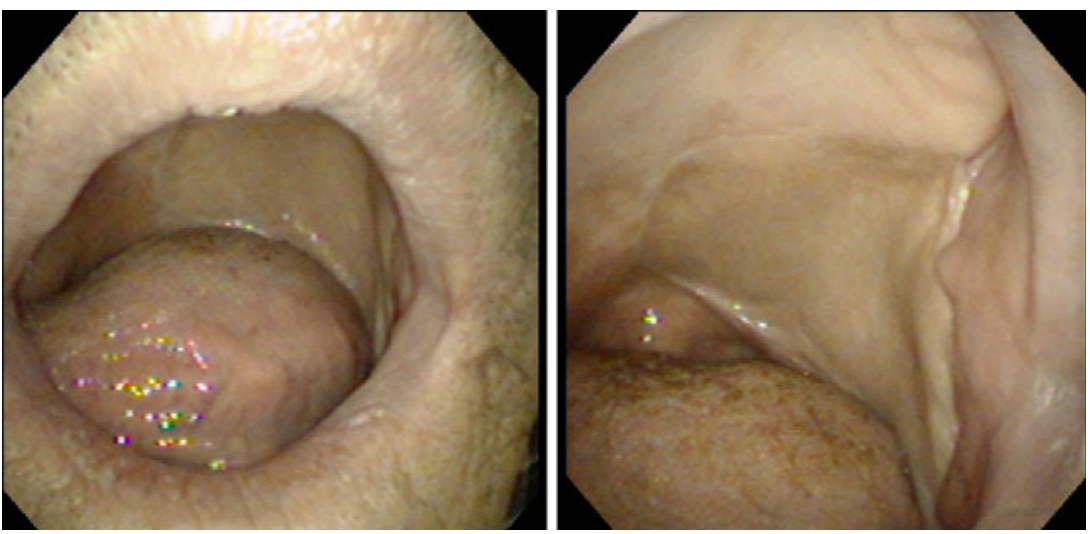
study, three reviewers independently graded the degree of permanent alopecia of 61 scalp regions in 26 patients who had undergone cranial irradiation greater than 12 months previously. Based on the dose-response curve in their paper, $60 \mathrm{~Gy}$ at $2 \mathrm{~Gy} /$ fraction would result in an $80 \%$ chance of permanent alopecia. In order to decrease the severity of acute and late side effects in our patient in light of his benign condition, we settled upon 50 Gy at 2 Gy/fraction.

Alternatively, a more comprehensive adjuvant radiation course could have been delivered, with 54-60 Gy given to the mucosal graft site plus margin as well as the ipsilateral neck. This would have resulted in increased dose to the ipsilateral parotid as well as a larger volume of higher dose the pharyngeal mucosa and tongue. Given that the patient was clinically disease-free 5 months out from resection for small primaries with low-risk pathologic features, the oncologic benefit would have likely been minimal.

Additionally, in light of his smoking history, we may have increased his risk for second malignancy with a more comprehensive radiation plan that included radiation exposure to the neck. Finally, the rationale for radiation in his case was palliation of sequelae (caused by hair growth) from his flap reconstruction, not oncologic management.

\section{Conclusion}

In a very select case, we were able to successfully treat a gentleman with unwanted and symptomatic palatal hair that was not amenable to traditional laser or electrolysis treatments. Advanced imaging, radiation planning, localization, and delivery techniques allowed us to minimize radiation side effects while achieving our goal of permanent alopecia.

Acknowledgments We acknowledge Gareth Smith for his assistance in formatting figures.

\section{References}

1. Hall RR, Pearce DJ, Brown T, McMichael AJ (2009) Unwanted palatal hair: a consequence of complex oropharyngeal reconstruction. J Dermatol Treat 20(3):149-151. doi:10.1080/09546630802562450

2. Badran D, Soutar DS, Robertson AG, Reid O, Milne EW, McDonald SW, Scothorne RJ (1998) Behavior of radial forearm skin flaps transplanted into the oral cavity. Clin Anat 11(6):379-389. doi:10.1002/ (SICI)1098-2353(1998)11:6<379::AID-CA3>3.0.CO;2-H

3. Haedersdal M, Wulf HC (2006) Evidence-based review of hair removal using lasers and light sources. J Eur Acad Dermatol Venereol: JEADV 20(1):9-20. doi:10.1111/j.1468-3083.2005.01327.x

4. Conroy FJ, Mahaffey PJ (2009) Intraoral flap depilation using the long-pulsed alexandrite laser. J Plast Reconstr Aesthet Surg: JPRAS 62(11):e421-e423. doi:10.1016/j.bjps.2008.03.051

5. Willey A, Torrontegui J, Azpiazu J, Landa N (2007) Hair stimulation following laser and intense pulsed light photo-epilation: review of 543 cases and ways to manage it. Lasers Surg Med 39(4):297301. doi:10.1002/1sm.20485

6. Lawenda BD, Gagne HM, Gierga DP, Niemierko A, Wong WM, Tarbell NJ, Chen GT, Hochberg FH, Loeffler JS (2004) Permanent alopecia after cranial irradiation: dose-response relationship. Int J Radiat Oncol Biol Phys 60(3):879-887. doi:10.1016/ j.ijrobp.2004.04.031 